\title{
Scedosporium Cerebral Abscesses After Extra-Corporeal Membrane Oxygenation
}

\author{
Hosam Al-Jehani, Marie-Christine Guiot, Carlos Torres, Judith Marcoux
}

\begin{abstract}
Background: Scedosporial infections are usually encountered in the immunocompromised patients. However, they are now emerging in the immunocompetent population and have an affinity for the central nervous system. They represent a therapeutic challenge, since they are highly resistant to most antifungal medications. Methods: We report the case of an immunocompetent patient with multiple cerebral abscesses secondary to Scedosporium apiospermum following extracorporeal membrane oxygenation (ECMO) and review the literature about this challenging cerebral infection. Results: A previously healthy 33-year-old male admitted to the hospital for a community-acquired pneumonia requiring ECMO subsequently developed multiple cerebral abscesses. He was empirically treated with caspofungin, which was changed to voriconazole once surgical aspiration revealed Scedosporium apiospermum. Despite multiple aspirations, decompressive craniectomy to alleviate intracranial pressure, and an appropriate antifungal agent, the patient did not survive this aggressive infection. Conclusion: Brain abscesses with Scedosporium apiospermum present a therapeutic challenge. High clinical suspicion leading to early appropriate antifungal therapy and combined surgical interventions might improve the prognosis.
\end{abstract}

RÉSUMÉ: Abcès cérébraux à Scedosporium après assistance respiratoire extra-corporelle. Contexte : Les infections à Scédosporium se rencontrent habituellement chez des patients immunocompromis. Cependant, on les rencontre maintenant dans la population immunocompétente et elles ont une affinité pour le système nerveux central. Leur traitement constitue un défi, étant donné qu'elles sont très résistantes à la plupart des médicaments antifongiques. Méthodes : Nous décrivons le cas d'un patient immunocompétent qui a présenté de multiples abcès cérébraux dus à Scedosporium apiospermum après assistance respiratoire extracorporelle (AREC) et nous revoyons la littérature sur cette infection cérébrale dont la prise en charge présente un défi. Résultats : Un homme de 33 ans sans antécédent pathologique a été hospitalisé pour une pneumonie extrahospitalière qui a nécessité une AREC. Il a par la suite présenté de multiples abcès cérébraux pour lesquels il a été traité de façon empirique par la caspofungine, puis par le voriconazole après que l'aspiration chirurgicale ait révélé la présence de Scédosporium apiospermum. Le patient est décédé malgré de multiples aspirations, une craniectomie décompressive pour diminuer la pression intracrânienne et l'administration d'un antifongique approprié. Conclusion : Les abcès cérébraux à Scédosporium apiospermum présentent un défi thérapeutique. Un haut degré de soupçon clinique motivant un traitement précoce par un antifongique approprié, combiné à la chirurgie, pourraient en améliorer le pronostic.

Can. J. Neurol. Sci. 2010; 37: 671-676

Scedosporial infections are emerging as highly resistant opportunistic infections. The clinical term 'scedosporiosis' is used for infections with both Scedosporium apiospermum, the anamorph (asexual state) of the ascomycete Pseudallescheria boydii, and Scedosporium prolificans. Usually encountered in the immunocompromised patients, experience is emerging in the immunocompetent population. ${ }^{1}$ Scedosporium species are often found in soil and contaminated water and are therefore sometimes associated with near-drowning events ${ }^{2-12}$ in immunocompetent hosts. The infections are highly resistant to most antifungal medications, including the new systemically active antifungal agents, such as triazoles and echinocandins. ${ }^{1}$ Involvement of the central nervous system creates an extra challenge as most antifungal agents do not readily penetrate the blood-brain barrier.

We report the case of an immunocompetent patient with multiple cerebral abscesses secondary to Scedosporium apiospermum, who did not survive despite an aggressive multimodality treatment approach. We also review the literature about this challenging cerebral infection.

\section{Case Report}

A 33-year-old Caucasian male, previously healthy and nonsmoker, was admitted with a suspicion of community-acquired pneumonia subsequent to a viral illness. On admission, the patient was febrile and severely hypoxic $\left(\mathrm{O}_{2} \mathrm{Sat}=80 \%\right)$. The chest radiograph showed severe bilateral infiltrates. The laboratory investigations revealed leukopenia (white blood cell count $=0.9 \times 10^{-9} / \mathrm{L}$ ) and neutropenia (neutrophile count $=0.4 \times$

From the Department of Neurosurgery (HAJ, JM), Department of Pathology (MCG), Montreal Neurological Institute and Hospital; Department of Neurosurgery (JM), Montreal General Hospital, McGill University, Montreal, Quebec; Department of Diagnostic Imaging (CT), The Ottawa Hospital, Civic Campus, Ottawa, Ontario, Canada.

Received October 7, 2009. Final Revisions Submitted March 23, 2010. Correspondence to: Judith Marcoux, Neurosurgery Department, McGill University, Montreal General Hospital, 1650 Cedar Avenue, Room: L7-524, Montreal, Quebec, H3G 1A4, Canada. 
$\left.10^{-9} / \mathrm{L}\right)$. The complete white blood cell count differential was as follow: lymphocytes $=0.3 \times 10^{-9} / \mathrm{L}$, monocytes $0.0 \mathrm{x}$ $10^{-9} / \mathrm{L}$; eosinophiles $=0.0 \times 10^{-9} / \mathrm{L}$; basophiles $=0.0 \times 10^{-9} / \mathrm{L}$; metamyelocytes $=0.1 \times 10^{-9} / \mathrm{L}$. The serum creatinine was 178 umol/L and lactate levels were $5.5 \mathrm{mmol} / \mathrm{L}$, indicating an impending renal failure. Glucose serum level was $5.2 \mathrm{mmol} / \mathrm{L}$ on admission and levels remained between 5.0 and $7.5 \mathrm{mmol} / \mathrm{L}$ throughout hospitalization. The patient did not respond to oxygenotherapy and BiPap (Bi-level Positive Airway Pressure) and had to be intubated within 24 hours of admission. The blood cultures from the sample taken at the time of admission revealed Streptococcus pneumoniae. He was treated with ceftriaxone and moxifloxacin. The patient then became hemodynamically unstable and required vasopressor therapy (norepinephrine). The renal function continued to deteriorate as well and the patient became anuric, which was treated with continuous veno venous hemodiafiltration. On Day 6, the oxygen saturation dropped to $65 \%$ despite nitric oxide therapy and oxygen supply of $100 \%$. The deteriorating respiratory function raised a suspicion of adult respiratory distress syndrome and the patient was treated with hydrocortisone sodium succinate (100 mg intravenously every eight hours) for 24 hours. Peripheral veno-venous extracorporeal membrane oxygenation (ECMO) was instituted which resulted in improvement of oxygenation.

The sedative agents that were given since the patient's admission could be alleviated and, on Day 12, the patient started to open his eyes spontaneously, obey commands, and nod appropriately (Glasgow coma scale (GCS) ${ }^{13}$ motor score of $6 / 6$ and eye opening of $4 / 4$; the patient was still intubated). On Day 14 , a line infection developed for which vancomycin was added to the treatment regimen and the ECMO was removed. On Day 20 , the patient was noticed to have a decreased movement of the

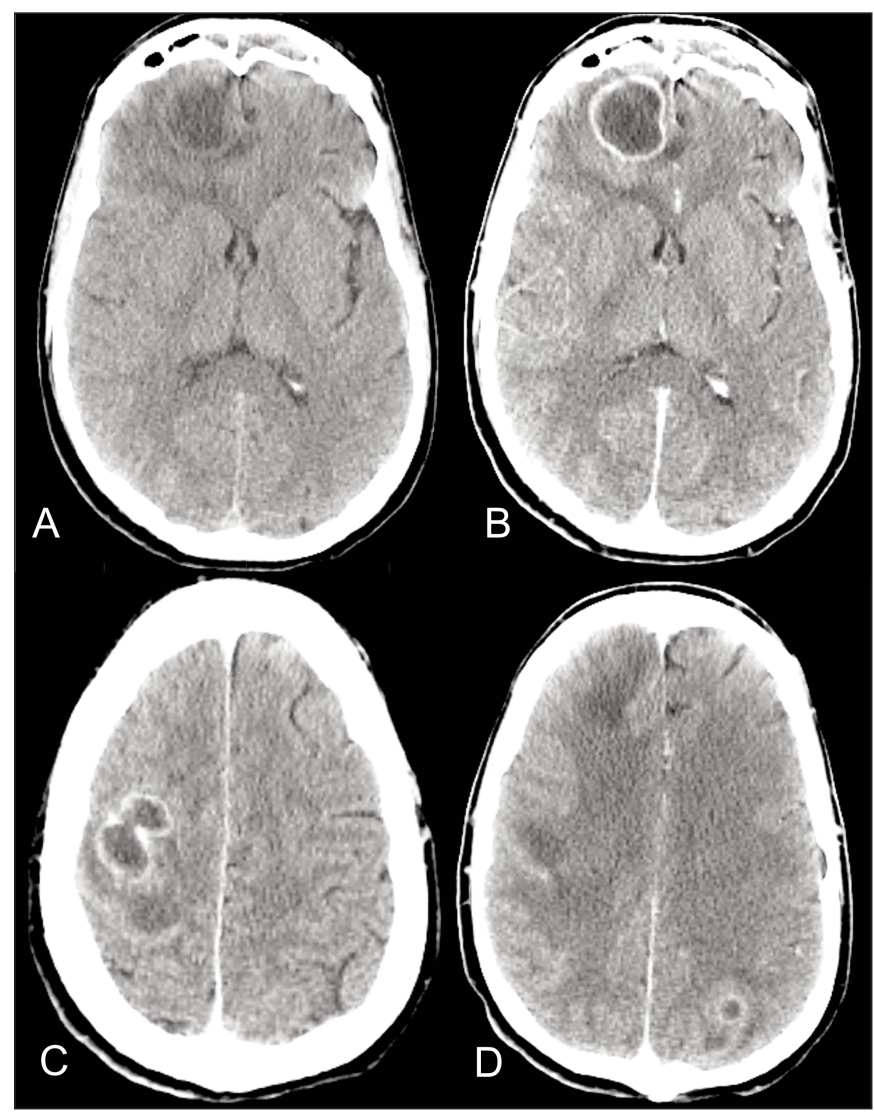

Figure 1: Plain (A) and contrast enhanced (B,C and D) head $C T$ showing multiple ring enhancing lesions with surrounding vasogenic edema, in both cerebral hemispheres.

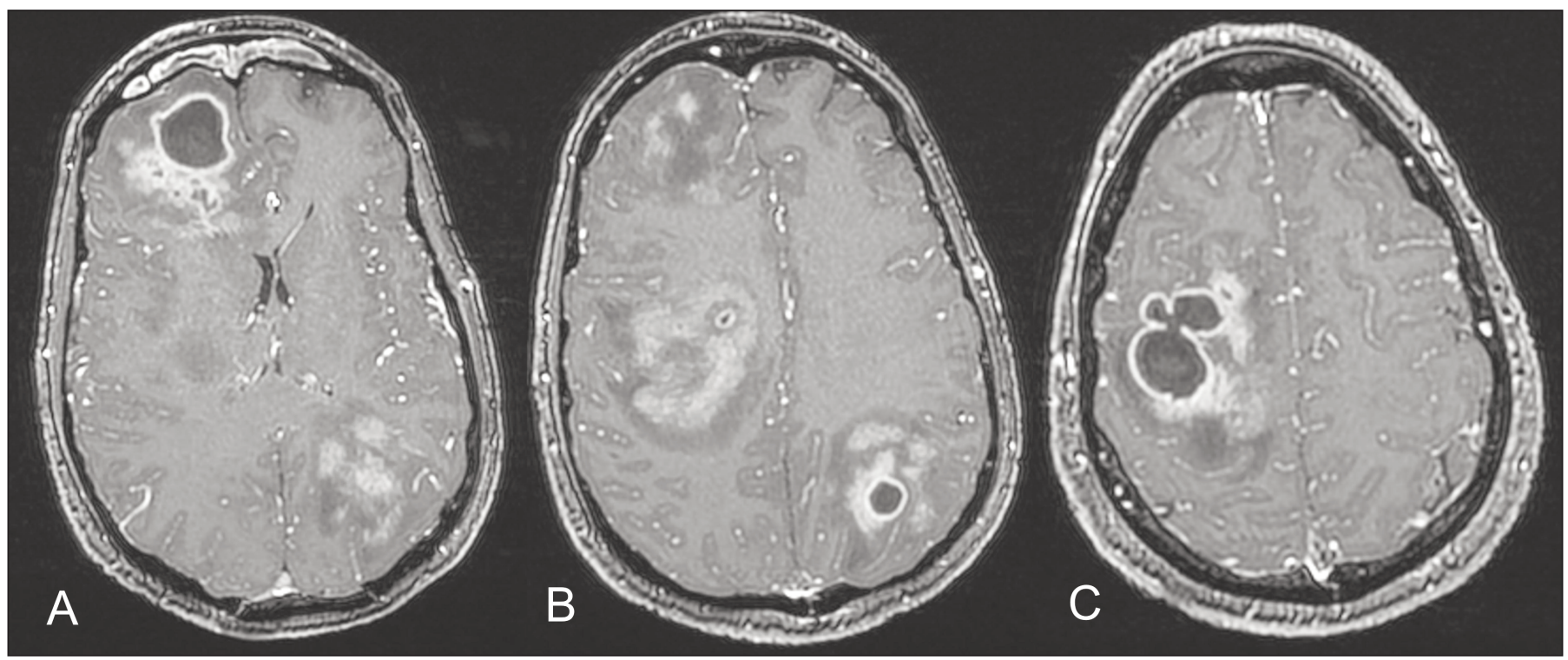

Figure 2: A Gadolinium enhanced MRI of the brain demonstrating multiple ring enhancing cerebral abscesses as well as surrounding areas of edema and cerebritis ( $A, B$ and $C$ ). 
left arm. On Day 21, a partial seizure involving the left side was noted as well. He was started on intravenous phenytoin and a computed tomogram (CT)-scan of the brain was performed. It demonstrated multiple brain abscesses with at least four ring enhancing lesions with surrounding vasogenic edema in both cerebral hemispheres (Figure 1). The patient was started empirically on caspofungin for possible fungal abscesses. On Day 22, percutaneous drainage of the largest lesion (right frontal) was performed with a small twist drill hole. However, the neurological condition continued to worsen. The patient became completely hemiplegic on the left side on Day 26. However, he could still open his eyes spontaneously and obey commands on the right side (motor score of the GCS motor score of $6 / 6$ and eye opening of 4/4). A brain magnetic resonance imaging (MRI) was performed which demonstrated multiple cystic mass lesions in the right frontal lobe, right paracentral region and left parietal lobe. The lesions showed peripheral enhancement after Gadolinium administration and measured between 2 and $25 \mathrm{~mm}$ in greater diameter. Also noticed were multiple small abscesses bilaterally and radiological evidence of diffuse meningitis

On Day 27, the preliminary findings from the percutaneous drainage were reported, which revealed presence of filamentous fungi. A stereotactic drainage was performed on the two largest lesions (right frontal and right parietal). The patient remained hemiplegic despite decreased size of abscesses. A repeat CT-scan

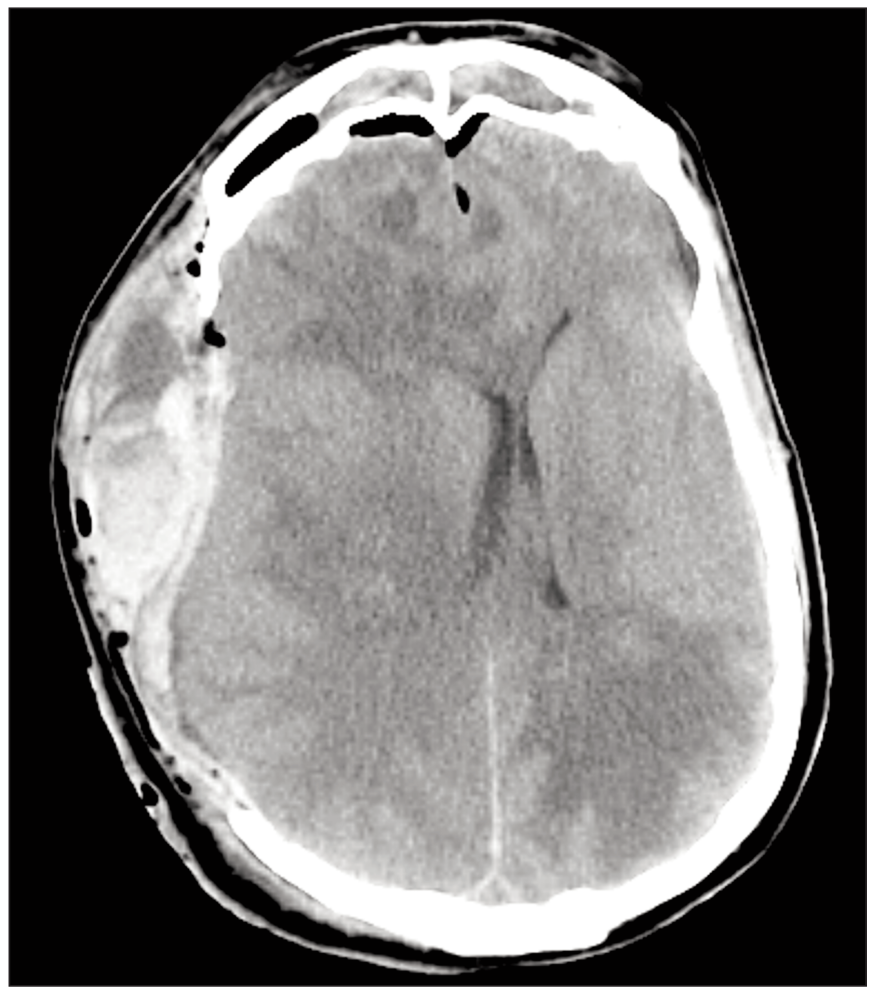

Figure 3: Post-operative non-enhanced CT head, showing an acute epidural hematoma in the right temporal region. demonstrated increased edema with an associated midline cerebral herniation of more than $5 \mathrm{~mm}$. Steroid therapy was instantly initiated to decrease mass effect, despite the presence of a fungal infection. Meanwhile the culture reports became clearer and suggested the presence of Pseudallescheria boydii. Voriconazole (400 mg oral every 12 hours) was added to the caspofungin (70 mg intravenously every day).

A bone marrow biopsy was performed on Day 29, which showed only a proliferative myeloid shift (most likely reactive) and no abnormal increase in blast or infiltration process. The patient's level of consciousness deteriorated (GCS score of 8T (Motor 5/6, Eye 2/4 and 1T-intubated) and an MRI of the brain was repeated, which showed a residual collection in the right frontal lobe along with more severe signs of cerebritis and increase in size of the left hemispheric abscess (Figure 2). A percutaneous drainage was repeated and $1 \mathrm{~mL}$ fluid was drained out. The final report from the culture showed Scedosporium apiospermum. The cerebritis and the edema continued to worsen, causing increasing mass effect. On Day 31, a decompressive craniectomy was performed on the right side to relieve the intracranial hypertension. Unfortunately, the surgery resulted in development of a large epidural hematoma (Figure 3 ). This hematoma was urgently evacuated but postoperatively, the patient showed minimal signs of consciousness with the pupils fixed and dilated. The next day, the pupils became sluggishly reactive and the patient exhibited rare movements to painful stimuli with his right upper extremity (decortication) (GCS 5) but no movement was noted on the left side. The condition remained unimproved over the next five days. A repeated CTscan demonstrated worsening of the fungal lesions. On Day 36 (five days post-craniectomy and herniation), the family members were consulted about patient's poor neurological condition and lack of response to treatment. The treatment was then terminated and comfort measures were started. The patient expired 30 minutes later.

An autopsy was performed to get further insight on the etiology. External examination of the brain showed a large bloody swelling in right frontal lobe. On coronal sections, bilateral uncal herniation with an obvious shift from right to left was noted. Multiple abscesses were also seen throughout the brain (right frontal lobe, left parietal lobe, right occipital lobe). Microscopic examination of different sections confirmed the diagnosis of fungal infection with the visualization of hyphae with Grocott stain (Figure 4). The main systemic finding was the presence of bilateral confluent bronchopneumonia, focally necrotizing with abscess formations. Microscopic examination of the lung suggested a bacterial origin for the lung abscesses; fungal stain was negative.

\section{DISCUSSION}

The literature reveals limited experience with cerebral scedosporiosis. Scedosporium apiospermum and Pseudallescheria boydii seem to have a high trophism for central nervous

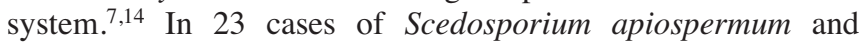
Pseudallescheria boydii infections reported by Katragkou et al. following near-drowning events, 21 involved the brain. ${ }^{8}$ It is more commonly found in immunocompromised hosts, ${ }^{14}$ like solid organ transplant patients ${ }^{15-18}$ or hematopoietic stem cell transplant patients, ${ }^{19}$ in patients with hematological malignancy ${ }^{20-22}$ or chronic granulomatous disease..$^{23}$ 


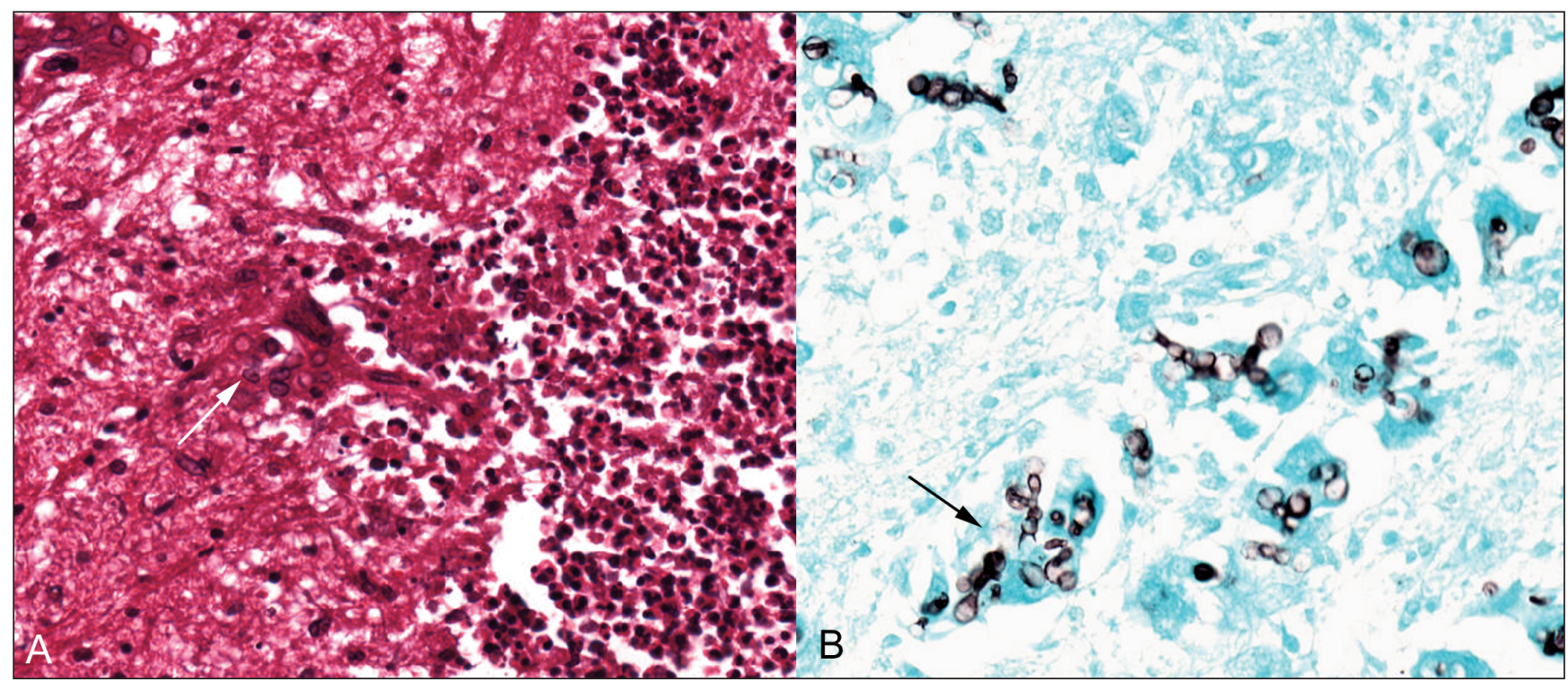

Figure 4: Hematoxylin and eosin stain (HE): Inflammatory infiltrate composed mainly of neutrophils (right half) and presence of fungal elements which faintly stain with HE (arrow). B: Groccot stain: Presence of branching hyphae (arrow).

In immunocompetent hosts, 24 out of 44 cases of Pseudallescheria boydii central nervous system infection were after near drowning events. ${ }^{7}$ The second commonest cause was trauma. ${ }^{7}$ In 12 cases of brain abscesses with Scedosporium apiospermum in immunocompetent hosts, nine were after neardrowning events, ${ }^{2-4,6,8-10,24,25}$ one following an infected foot injury with Scedosporium apiospermum, ${ }^{26,27}$ one following chronic suppurative otitis in a child, ${ }^{28}$ one in a previously healthy woman who developed HELLP (Hemolytic anemia, Elevated Liver enzymes and Low Platelet count) syndrome and needed ECMO as well. ${ }^{29}$ One case of chronic meningitis in a woman with uncontrolled diabetes has also been reported. There never was intracerebral involvement in that case however. ${ }^{30}$

We described a case of cerebral abscess secondary to Scedosporium apiospermum. This patient was a previously healthy young immunocompetent male. His presentation of bronchopneumonia and fulminant course with severe sepsis and florid pulmonary infiltrate is unusual but bone marrow aspiration and blood screening tests failed to demonstrate immunodeficiency. The patient however had neutropenia upon admission, severe sepsis, and received aggressive antibiotherapy, increasing his risks of hematogenous spread of opportunistic fungal infection into the brain parenchyma. With the progressive deterioration of his pulmonary status; the patient was placed on ECMO. The use of ECMO could be a precipitant of cerebral fungal infection, as the filtering action of the pulmonary vascular bed is bypassed. Extracorporeal membrane oxygenation has previously been associated with cerebral fungal infections. ${ }^{31-35}$ The concept of vascular bypass predisposing to cerebral fungal infections is further supported by the reported case of such an infection in a heart transplant patient who had an ECMO followed by a right ventricular assist device. ${ }^{36}$ It was suggested that the use of steroids as a part of near drowning treatment protocols and the hypoxic state at the time of the event ${ }^{14}$ could be a contributing factor to the cerebral dissemination of this infection.

The management of cerebral scedosporiosis is extremely challenging. The challenges in our case included severe cerebritis and multiple very small abscesses, not amenable to surgical drainage. The decompressive craniectomy was performed in an attempt to relieve the building intracranial hypertension. The aim was to improve cerebral autoregulation and cerebral blood flow and, hence, the delivery of systemic antifungal medication to help in controlling the infectious focus. This was not found to be helpful and a complication from the procedure further complicated the clinical course. While the surgical outcomes have usually been poor in treatment of scedosporiosis, ${ }^{37}$ there is a recent report ${ }^{10}$ of a successful treatment of this infection with an emergency craniotomy followed by long-term voriconazole therapy. The patient had no relapse of cerebral infection after 18 months but remained in a minimally conscious state. Another case of a young child successfully treated with repeated aspirations and voriconazole was described. ${ }^{4}$ Of note is that this particular case seemed to have followed a much less aggressive course, being diagnosed three months after the near-drowning accident because of persistently poor neurological exam but without acute deterioration as in our patient, and receiving voriconazole only three months after the initial diagnosis. The child remained with significant neurological sequelae. Another case in an adult patient with diabetes and acute lymphoblastic leukemia was successfully treated with posaconazole. ${ }^{20}$ Surgery had failed to make any improvement, even if the images showed well circumscribed lesions, contrary to our case with significant cerebritis. 
Voriconazole is a synthetic second-generation, broadspectrum triazole derivative of fluconazole. It has anti-fungal properties by inhibiting the cytochrome P450 (CYP)-dependent enzyme 14-alpha-sterol demethylase, thereby disrupting the cell membrane and halting fungal growth. ${ }^{38}$ Voriconazole is a strong anti-fungal agent that demonstrated clinical efficacy, with good blood brain barrier penetration and a tolerable profile even for a prolonged course..$^{10}$ It had a response rate of $54 \%$ against Scedosporium apiospermum in one large study and was well tolerated ${ }^{39}$ It has been used to treat Scedosporium apiospermum infections in many different sites, including cutanenous infections, ${ }^{40-45}$ endophtalmitis, ${ }^{46,47}$ osteomyelitis in a child, ${ }^{48}$ and disseminated infection in a transplant patient ${ }^{49}$ among reported cases.

Our case might justify early specific genetic diagnostic testing to facilitate and fasten the diagnosis. An early accurate diagnosis can help better direct the antifungal therapy, promoting a more successful outcome. Conventional phenotypic methods have low sensitivity and specificity. Molecular techniques can give an accurate phylogenetic delineation of species, and rapid identification of pathogen strains. ${ }^{50}$ It has been suggested to use panfungal polymerase chain reaction (PCR) in high-risk patients (eg: neutropenic patients) with evidence of fungal infection. ${ }^{8}$ Spiess et $\mathrm{al}^{51}$ have developed a DNA microarray combining multiplex PCR and consecutive DNA microarray hybridization, enabling them to detect DNA from 14 different fungal pathogens. There are currently five different methods (multilocus enzyme electrophoresis, random amplification of polymorphic DNA, PCR finger-printing, restriction fragment length polymorphism, amplified fragment-length polymorphism, intergenic spacer region PCR used for genotyping of Scedosoprium species, with advantages and drawbacks for each of them. A new one, multilocus Sequence Typing, is currently being developed. ${ }^{50}$

Cerebral fungal infection is associated with a poor prognosis overall. In a series of 18 patients with cerebral mycosis (including two with Scedosporium apiospermum) in a tertiary care center, 12 died, two were lost to follow-up, and the four remaining patients remained with severe neurological sequelae..$^{52}$ Some pathogens, like Aspergillus species and Zygomycetes carry a worse prognosis then Cryptococcus or Candida species due to their angioinvasiveness proprieties. ${ }^{53,54}$ The overall mortality rate for CNS aspergillosis is as high as $90 \% .{ }^{55}$ Survival is rare in immunocompromise patients. ${ }^{56}$ However, a good outcome can be achieved in immunocompetent hosts with a survival rate reported between 40 and $80 \% .{ }^{54}$ In two recent case series, all six reported patients achieved a good recovery. ${ }^{57,58}$ In cerebral infections with Pseudallescheria boydii, only one survived in a series of 11 transplant patients. ${ }^{16}$ A review of cases in the literature for Pseudallesheria Boydii had nine survivors for 19 deaths ${ }^{59}$ Another review reported a mortality rate of $74 \%$ for Pseudallescheria boydii. ${ }^{7}$ For Scedosporium apiospermum brain infection, in 12 cases reported in immunocompetent hosts since 1985 , nine succumbed to the disease, $, 3,3,6,8,9,12,24,26-29$ two survived with significant neurological sequelae $e^{4,10}$ and only one had a favorable outcome..$^{25}$

\section{Conclusion}

Brain abscesses caused by Scedosporium apiospermum are rare and present a therapeutic challenge. Clinical suspiscion of the diagnosis even in immunocompetent patients may lead to a very early appropriate antifungal therapy. Combined with timely surgical interventions, it might improve the prognosis of patients with cerebral scedosporiosis.

\section{REFERENCES}

1. Rodriguez-Tudela JL, Berenguer J, Guarro J, et al. Epidemiology and outcome of Scedosporium prolificans infection, a review of 162 cases. Med Mycol. 2008;1:1-12.

2. Baudrillard JC, Rousseaux P, Lerais JM, et al. Fungal mycotic aneurysms and multiple cerebral abscesses caused by Scedosporium apiospermum. A propos of a case with review of the literature. J Radiol. 1985;66(4):321-6.

3. Buzina W, Feierl G, Haas D, et al. Lethal brain abscess due to the fungus Scedosporium apiospermum (teleomorph Pseudallescheria boydii) after a near-drowning incident: case report and review of the literature. Med Mycol. 2006;44(5):473-7.

4. Chakraborty A, Workman MR, Bullock PR. Scedosporium apiospermum brain abscess treated with surgery and voriconazole. Case report. J Neurosurg. 2005;103 1 Suppl:83-7.

5. Dworzack DL, Clark RB, Borkowski WJ Jr, et al. Pseudallescheria boydii brain abscess: association with near-drowning and efficacy of high-dose, prolonged miconazole therapy in patients with multiple abscesses. Medicine (Baltimore). 1989;68(4): 218-24.

6. Gari M, Fruit J, Rousseaux P, et al. Scedosporium (Monosporium) apiospermum: multiple brain abscesses. Sabouraudia. 1985;23 (5):371-6.

7. Kantarcioglu AS, Guarro J, de Hoog GS. Central nervous system infections by members of the Pseudallescheria boydii species complex in healthy and immunocompromised hosts: epidemiology, clinical characteristics and outcome. Mycoses. 2008;51(4):275-90.

8. Katragkou A, Dotis J, Kotsiou M, et al. Scedosporium apiospermum infection after near-drowning. Mycoses. 2007;50 (5):412-21.

9. Kowacs PA, Soares Silvado CE, Monteiro de Almeida S, et al. Infection of the CNS by Scedosporium apiospermum after near drowning. Report of a fatal case and analysis of its confounding factors. J Clin Pathol. 2004;57(2):205-7.

10. Leechawengwongs $\mathrm{M}$, Milindankura $\mathrm{S}$, Liengudom $\mathrm{A}$, et al. Multiple Scedosporium apiospermum brain abscesses after neardrowning successfully treated with surgery and long-term voriconazole: a case report. Mycoses. 2007;50(6):512-6.

11. Mursch K, Trnovec S, Ratz H, et al. Successful treatment of multiple Pseudallescheria boydii brain abscesses and ventriculitis/ependymitis in a 2-year-old child after a neardrowning episode. Childs Nerv Syst. 2006;22(2):189-92.

12. Rüchel R, Wilichowski E. Cerebral Pseudallescheria mycosis after near-drowning. Mycoses. 1995;38(11-12):473-5.

13. Teasdale G, Jennett B. Assessment of coma and impaired consciousness: a practical scale. Lancet. 1974;2(7872):81-4.

14. Cortez KJ, Roilides E, Quiroz-Telles F, et al. Infections caused by Scedosporium spp. Clin Microbiol Rev. 2008 Jan;21(1):157-97.

15. Alsip SG, Cobbs CG. Pseudallescheria boydii infection of the central nervous system in a cardiac transplant recipient. South Med J. 1986;79(3):383-4.

16. Castiglioni B, Sutton DA, Rinaldi MG, et al. Pseudallescheria boydii (Anamorph Scedosporium apiospermum). Infection in solid organ transplant recipients in a tertiary medical center and review of the literature. Medicine (Baltimore). 2002;81(5): 333-48.

17. Luu KK, Scott IU, Miller D, et al. Endogenous Pseudallescheria boydii endophthalmitis in a patient with ring-enhancing brain lesions. Ophthalmic Surg Lasers. 2001;32(4):325-9. 
18. Satirapoj B, Ruangkanchanasetr $\mathrm{P}$, Treewatchareekorn S, et al. Pseudallescheria boydii brain abscess in a renal transplant recipient: first case report in Southeast Asia. Transplant Proc. 2008;40(7):2425-7.

19. Safdar A, Papadopoulos EB, Young JW. Breakthrough Scedosporium apiospermum (Pseudallescheria boydii) brain abscess during therapy for invasive pulmonary aspergillosis following high-risk allogeneic hematopoietic stem cell transplantation. Scedosporiasis and recent advances in antifungal therapy. Transpl Infect Dis. 2002;4(4):212-7.

20. Mellinghoff IK, Winston DJ, Mukwaya G, et al. Treatment of Scedosporium apiospermum brain abscesses with posaconazole. Clin Infect Dis. 2002;34(12):1648-50.

21. Pagano L, Caira M, Falcucci P, et al. Fungal CNS infections in patients with hematologic malignancy. Expert Rev Anti Infect Ther. 2005;3(5):775-85.

22. Yoo D, Lee WH, Kwon-Chung KJ. Brain abscesses due to Pseudallescheria boydii associated with primary non-Hodgkin's lymphoma of the central nervous system: a case report and literature review. Rev Infect Dis. 1985;7(2):272-7.

23. Bhat SV, Paterson DL, Rinaldi MG, et al. Scedosporium prolificans brain abscess in a patient with chronic granulomatous disease: successful combination therapy with voriconazole and terbinafine. Scand J Infect Dis. 2007;39(1):87-90.

24. Durieu I, Parent M, Ajana F, et al. Monosporium apiospermum meningoencephalitis: a clinico-pathological case. J Neurol Neurosurg Psychiatry. 1991;54(8):731-3.

25. Garzoni C, Emonet S, Legout L, et al. Atypical infections in tsunami survivors. Emerg Infect Dis. 2005;11:1591-3.

26. Horré R, Feil E, Stangel AP, et al. Scedosporiosis of the brain with fatal outcome after traumatizatio of the foot. Case report. Mycoses. 2000;43 Suppl 2:33-6.

27. Pennekamp PH, Diedrich O, Zhou H, et al. Foot injury as a rare cause of scendosporiosis with fetal outcome. Unfallchirurg. 2003;106(10):865-8.

28. Acharya A, Ghimire A, Khanal B, et al. Brain abscess due to Scedosporium apiospermum in a non immunocompromised child. Indian J Med Microbiol. 2006;24(3):231-2.

29. Riddell J 4th, Chenoweth CE, Kauffman CA. Disseminated Scedosporium apiospermum infection in a previously healthy woman with HELLP syndrome. Mycoses. 2004;47(9-10):442-6.

30. Danaher PJ, Walter EA. Successful treatment of chronic meningitis caused by Scedosporium apiospermum with oral voriconazole. Mayo Clin Proc. 2004;79(5):707-8.

31. Brüggemann RJ, Antonius T, Heijst A, et al. Therapeutic drug monitoring of voriconazole in a child with invasive aspergillosis requiring extracorporeal membrane oxygenation. Ther Drug Monit. 2008;30(6):643-6.

32. Minette MS, Ibsen LM. Survival of candida sepsis in extracorporeal membrane oxygenation. Pediatr Crit Care Med. 2005;6(6): 709-11.

33. O'Neill JM, Schutze GE, Heulitt MJ, et al. Nosocomial infections during extracorporeal membrane oxygenation. Intensive Care Med. 2001;27(8):1247-53.

34. Sachweh JS, Tiete AR, Fuchs A, et al. Efficacy of extracorporeal membrane oxygenation in a congenital heart surgery program. Clin Res Cardiol. 2007;96(4):204-10.

35. Sander A, Beyer U, Amberg R. Systemic Fusarium oxysporum infection in an immunocompetent patient with an adult respiratory distress syndrome (ARDS) and extracorporal membrane oxygenation (ECMO). Mycoses. 1998;41(3-4): $109-11$.

36. Eschertzhuber S, Velik-Salchner C, Hoermann $\mathrm{C}$, et al. Caspofungin-resistant Aspergillus flavus after heart transplantation and mechanical circulatory support: a case report. Transpl Infect Dis. 2008;10(3):190-2.

37. Tang SC, Huang SJ, Chiu MJ, et al. Impaired cerebral autoregulation in a case of severe acute encephalitis. J Formos Med Assoc. 2007;106 Suppl 2:S7-12.

38. Scott LJ, Simpson D. Voriconazole: a review of its use in the management of invasive fungal infections. Drugs. 2007;67(2):269-98.
39. Troke P, Aguirrebengoa K, Arteaga C, et al. Global Scedosporium Study Group. Treatment of scedosporiosis with voriconazole: clinical experience with 107 patients. Antimicrob Agents Chemother. 2008;52(5): 1743-50.

40. Matsumoto Y, Oh-I T, Nagai A, et al. Case of cutaneous Scedosporium apiospermum infection successfully treated with voriconazole. J Dermatol. 2009;36(2):98-102.

41. Stur-Hofmann K, Stos S, Saxa-Enenkel M, et al. Primary cutaneous infection with Scedosporium apiospermum successfully treated with voriconazole. Mycoses. Epub 2009 Nov 18.

42. Al-Badriyeh D, Leung L, Davies GE, et al. Successful salvage treatment of Scedosporium apiospermum keratitis with topical voriconazole after failure of natamycin. Ann Pharmacother. 2009;43(6): 1139-42.

43. Bunya VY, Hammersmith KM, Rapuano CJ, et al. Topical and oral voriconazole in the treatment of fungal keratitis. Am J Ophthalmol. 2007;143(1):151-3

44. Jhanji V, Yohendran J, Constantinou M, et al. Scedosporium scleritis or keratitis or both: case series. Eye Contact Lens. 2009; 35(6):312-5.

45. Nochez Y, Arsene S, Le Guellec C, et al. Unusual pharmacokinetics of intravitreal and systemic voriconazole in a patient with Scedosporium apiospermum endophthalmitis. J Ocul Pharmacol Ther. 2008;24(1):87-90.

46. Chen FK, Chen SD, Tay-Kearney ML. Intravitreal voriconazole for the treatment of endogenous endophthalmitis caused by Scedosporium apiospermum. Clin Experiment Ophthalmol. 2007;35(4):382-5.

47. Zarkovic A, Guest S. Scedosporium apiospermum traumatic endophthalmitis successfully treated with voriconazole. Int Ophthalmol. 2007;27(6):391-4.

48. Stripeli F, Pasparakis D, Velegraki A, et al. Scedosporium apiospermum skeletal infection in an immunocompetent child. Med Mycol. 2009;47(4):441-4.

49. Rogasi PG, Zanazzi M, Nocentini J, et al. Disseminated Scedosporium apiospermum infection in renal transplant recipient: long-term successful treatment with voriconazole: a case report. Transplant Proc. 2007 Jul-Aug;39(6):2033-5.

50. Harun A, Perdomo H, Gilgado F, et al. Genotyping of Scedosporium species: a review of molecular approaches. Med Mycol. 2009;47(4):406-14.

51. Spiess B, Seifarth W, Hummel M, et al. DNA microarray-based detection and identification of fungal pathogens in clinical samples from neutropenic patients. J Clin Microbiol. 2007;45 (11):3743-53

52. Raparia K, Powell SZ, Cernoch P, et al. Cerebral mycosis: 7-year retrospective series in a tertiary center. Neuropathology. Epub 2009 Oct 21

53. Redmond A, Dancer C, Woods ML. Fungal infections of the central nervous system: a review of fungal pathogens and treatment. Neurol India. 2007;55(3):251-9.

54. Shamim MS, Siddiqui AA, Enam SA, et al. Craniocerebral aspergillosis in immunocompetent hosts: surgical perspective. Neurol India. 2007;55(3):274-81.

55. Denning DW, Stevens DA. Antifungal and surgical treatment of invasive aspergillosis: review of 2121 published cases [published correction appears in Rev Infect Dis. 1991;13:345]. Rev Infect Dis. 1990;12:1147-201.

56. Nadkarni T, Goel A. Aspergilloma of the brain: an overview. J Postgrad Med. 2005;51:37-41.

57. Louati I, Zaouali J, Azzouz O, et al. Cerebral aspergillosis in immunocompetent patients: three case reports. Rev Neurol (Paris). 2009;165(11):957-61

58. Srinivasan US. Intracranial aspergilloma in immunocompetent patients successfully treated with radical surgical intervention and antifungal therapy: case series. Ann Acad Med Singapore. 2008;37(9):783-7.

59. Nesky MA, McDougal EC, Peacock Jr JE. Pseudallescheria boydii brain abscess successfully treated with voriconazole and surgical drainage: case report and literature review of central nervous system pseudallescheriasis. Clin Infect Dis. 2000;31(3):673-7. 